\title{
2D phaseless super-resolution
}

\section{Myung Cho, Christos Thramboulidis, Babak Hassibi, Weiyu Xu}

Myung Cho, Christos Thramboulidis, Babak Hassibi, Weiyu Xu, "2D phaseless super-resolution," Proc. SPIE 10394, Wavelets and Sparsity XVII, 103941H (24 August 2017); doi: 10.1117/12.2275409

EDent: SPIE Optical Engineering + Applications, 2017, San Diego, California, United States 


\title{
2D Phaseless Super-resolution
}

\author{
Myung Cho ${ }^{a}$, Christos Thrampoulidis ${ }^{\mathrm{b}}$, Babak Hassibic ${ }^{\mathrm{c}}$, and Weiyu $\mathrm{Xu}^{\mathrm{a}}$ \\ aDept. of ECE, University of Iowa, Iowa City, IA, 52242, USA \\ ${ }^{b}$ LIDS, Massachusetts Institute of Technology, Cambridge, MA, 02139, USA \\ 'Dept. of EE, California Institute of Technology, Pasadena, CA, 91125, USA
}

\begin{abstract}
In phaseless super-resolution, we only have the magnitude information of continuously-parameterized signals in a transform domain, and try to recover the original signals from these magnitude measurements. Optical microscopy is one application where the phaseless super-resolution for $2 \mathrm{D}$ signals arise. In this paper, we propose algorithms for performing phaseless super-resolution for 2D or higher-dimensional signals, and investigate their performance guarantees.
\end{abstract}

Keywords: super-resolution, phase retrieval, microscopy, optical microscopy, magnitude, mask, filter

\section{INTRODUCTION}

In the field of science and engineering, the research on overcoming or mitigating the limitations of measurement tools has been widely conducted. For example, in 2014, the Nobel Prize in Chemistry was awarded to the researchers for the development of super-resolution fluorescence microscopy. ${ }^{1}$ Especially, in optical microscopy, a target object of interest can be observed or investigated by resolving the elements of structural features with light sources. Because of its usability to the biological structure of living cell unlike electron microscopy, optical microscopy has been considered as an important measurement tool in various fields such as biology and medicine. However, due to physical limitations including Abbe's diffraction limit, ${ }^{2}$ obtaining high resolved images beyond the physical limitation by using optical microscopy is still on-going research. In addition, due to the nature of light source, it is hard to measure phase information rather than magnitude information from optical microscopy. Hence, the research on recovering a signal of interest or observing an object of interest by using limited measurement data, e.g., only magnitude information and only low frequency information, have been studied.

Super-resolution and phase retrieval are the well known signal processing problems from limited measurement data. In the super-resolution, low frequency measurement data including phase as well as magnitude information are considered to recover a signal of interest with unknown high frequency information. The phase retrieval aims at the recovery of a signal of interest up to phase only with the magnitude information of the sampled signal. For optical microscopy, the limited measurement data can be low frequency magnitude information of the sampled signal. Therefore, the conventional super-resolution and phase retrieval theorems cannot be directly applicable to the optical microscopy.

Phaseless super-resolution combines the two problems of super-resolution and of phase-retrieval and seeks to recover a signal from magnitude measurements of only low-frequencies. Hence, due to the extremely limited measurement data and type including only low frequency and only magnitude information, the phaseless super-resolution is a well ill-posed and a challenging problem. This phaseless super-resolution problem is firstly introduced by Jaganathan et al. for a signal in 1D which is expressed as sum of Dirac Delta functions located in the discrete domain, i.e., on the grid. ${ }^{3}$ By using lifting method, the authors proposed the Semidefinite Programming (SDP) formulation for the phaseless super-resolution problem in the discrete domain and showed that one could recover a signal of interest in 1D from only the magnitude of low frequency information. Later, Cho et al. dealt with the phaseless super-resolution problem for an 1D signal in the continuous domain, i.e., off the

Further author information: (Send correspondence to Myung Cho)

E-mail: myung-cho@uiowa.edu

Wavelets and Sparsity XVII, edited by Yue M. Lu, Dimitri Van De Ville, Manos Papadakis, Proc. of SPIE Vol. 10394, 103941H · @ 2017 SPIE · CCC code: 0277-786X/17/\$18 · doi: 10.1117/12.2275409 
grid. ${ }^{4}$ In order to handle the nature of the continuous signal domain, Cho et al. introduced the squared atomic norm minimization and derived the SDP formulation of the squared atomic norm minimization which is called as Phaseless Atomic Norm Minimization (PANM).

The previous research on the phaseless super-resolution are targeting the recovery of a signal of interest in 1D. For practical applications such as microscopy, X-ray crystallography, and medical devices, dealing with signals in $2 \mathrm{D}$ or higher dimension is of great interest due to the nature of the original signal in high dimension and the wide range of applications. The super-resolution problem considering a signal in 2D or higher dimension was studied by Xu et al. ${ }^{5}$ and Chi et al. ${ }^{6}$ In particular, Xu et al. derived the precise SDP formulation of the Atomic Norm Minimization (ANM) for 2D or higher-dimensional signals by using positive trigonometric polynomial, ${ }^{5}$ while Chi et al. introduced an approximate SDP formulation to solve the ANM. ${ }^{6}$

The contribution of this paper is two-fold. This paper firstly describes the phaseless super-resolution problem for $2 \mathrm{D}$ or higher-dimensional signals in the continuous domain, which is uninvestigated research area. Secondly, from the phaseless measurement data, we propose the SDP formulation of PANM for 2D or higher-dimensional signals. This paper can be a bridge between theories and practical applications including optical microscopy and $\mathrm{x}$-ray crystallography to improve the resolution of those applications.

The rest of the paper is organized as follows. Section 2 describes the problem setting including the equation for $2 \mathrm{D}$ or higher-dimensional signals in the continuous domain. In Section 3, the super-resolution problem and ANM for 2D or higher dimensional signals are introduced. In Section 4, we propose the PANM for 2D or higherdimensional signals and its SDP formulation to solve the PANM. Finally, in Section 5, we demonstrate the successful recovery of a signal from low frequency magnitude measurement data by using the SDP formulation for the PANM.

Notations: We denote the sets of complex numbers and natural numbers as $\mathbb{C}$ and $\mathbb{N}$ respectively. We reserve calligraphic uppercase letters for index sets, e.g., $\mathcal{N} .|\mathcal{N}|$ represents the cardinality of the index set $\mathcal{N}$. We use the superscripts $*, T$, and $H$ to denote conjugate, transpose, and conjugate transpose respectively. We reserve $i$ for the imaginary number, i.e., $i^{2}=-1$. When we denote a sampled signal in the frequency domain as $x[\boldsymbol{f}]$, we represent its space domain signal as $\hat{x}(\boldsymbol{t})$. For a ground truth signal, we use the superscript $o$, e.g., $x^{o}$. If we use an index set as the subscript of a vector or a tensor, it represents the partial vector or the partial tensor over the index set. $\vec{x}_{j}$ denotes the $j$-th element of a vector $\vec{x}$.

\section{PROBLEM SETTING}

Let us consider a $d$-dimensional signal $\hat{x}^{o}(\boldsymbol{t})$, where $d \geq 2$ and $\operatorname{tin}[0,1]^{d}$. The signal $\hat{x}^{o}(\boldsymbol{t})$ that we are interested in is expressed as a sum of Dirac delta functions as follows:

$$
\hat{x}^{o}(\boldsymbol{t})=\sum_{j=1}^{s} c_{j}^{o} \delta\left(\boldsymbol{t}-\boldsymbol{t}_{j}^{o}\right)
$$

where $\boldsymbol{t}_{j}^{o} \in[0,1]^{d}, \delta(\boldsymbol{t})$ is the $d$-dimensional Dirac delta function, $c_{j}^{o}=\left|c_{j}^{o}\right| e^{i \phi_{j}^{o}},\left|c_{j}^{o}\right|>0$, and $\phi_{j}^{o} \in[0,2 \pi)$. Its Fourier transform $x^{o}[\boldsymbol{f}]$ is a sum of s complex exponentials as follows:

$$
x^{o}[\boldsymbol{f}]=\sum_{j=1}^{s} c_{j}^{o} e^{i 2 \pi \boldsymbol{f}^{T} \boldsymbol{t}_{j}^{o}}=\sum_{j=1}^{s}\left|c_{j}\right| a\left(\boldsymbol{t}_{j}^{o}, \phi_{j}^{o}\right)[\boldsymbol{f}], \boldsymbol{f} \in \mathcal{N} .
$$

The index set $f$ is defined as $\prod_{p=1}^{d}\left\{f \mid 0 \leq f \leq n_{p}-1\right\}$ to represent the set of frequency indices of the signal, where $n_{p} \in \mathbb{N}, 1 \leq p \leq d$, and $|\mathcal{N}|=\prod_{p=1}^{d} n_{p}$. We set $n_{p}=n$, for all $p=1,2, \ldots, d$, for the sake of simplicity. $a\left(\boldsymbol{t}_{j}, \phi_{j}\right) \in \mathcal{A} \subset \mathbb{C}^{|\mathcal{N}|}$ is temporal or spatial atom vector, where $\mathcal{A}$ is the set of all temporal or spatial atoms. For a specific frequency $\boldsymbol{f}, a\left(\boldsymbol{t}_{j}, \phi_{j}\right)[\boldsymbol{f}]=e^{i\left(2 \pi \boldsymbol{t}_{j}^{T} \boldsymbol{f}+\phi_{j}\right)}$, where $\boldsymbol{t}_{j}=\left(t_{j, 1}, t_{j, 2}, \ldots, t_{j, d}\right)^{T} \in[0,1]^{d}$.

Our goal here is recovering the $d$-dimensional signal introduced in (2.1) including $c_{j}^{o}$ and $\boldsymbol{t}_{j}^{o}$ for all $j=1, \ldots, s$ from only low frequency magnitude information. Since the ground truth signal $\hat{x}^{o}(\boldsymbol{t})$ is in the continuous domain, we consider the ANM for a multidimensional signal. Hence, in the next section, we will review the ANM for a multidimensional signal, and then, introduce how to adapt the magnitude frequency information in the ANM formulation. 


\section{ATOMIC NORM MINIMIZATION FOR MULTIDIMENSIONAL SIGNALS}

In this section, we review how ANM framework leads to an SDP formulation for recovering a continuous multidimensional signal as in (2.1) from its Fourier measurements. ${ }^{5}$

The atomic norm $\|\cdot\|_{\mathcal{A}}$ of a $d$-dimensional signal of the form (2.2) is defined as follows:

$$
\|x\|_{\mathcal{A}}=\inf \left\{\sum_{j}\left|c_{j}\right|: x[\boldsymbol{f}]=\sum_{j}\left|c_{j}\right| a\left(\boldsymbol{t}_{j}, \phi_{j}\right)[\boldsymbol{f}], \begin{array}{l}
\boldsymbol{t}_{j} \in[0,1]^{d} \\
\phi_{j} \in[0,2 \pi)
\end{array}\right\} .
$$

For two tensors $q$ and $x$, let us denote the vectorization of $q$ and $x$ as $\vec{q}$ and $\vec{x}$ respectively, and the inner product between them as $\langle q, x\rangle=\vec{x}^{H} \vec{q}$. We define the real part of the inner product as $\langle q, x\rangle_{\mathbb{R}}$. The dual norm is then expressed as follows:

$$
\|q\|_{\mathcal{A}}^{*}=\sup _{\|x\|_{\mathcal{A}} \leq 1}\langle q, x\rangle_{\mathbb{R}}=\sup _{\boldsymbol{t} \in[0,1]^{d}}|\langle q, a(\boldsymbol{t}, 0)\rangle| .
$$

With the definition of the atomic norm, the ANM for a $d$-dimensional signal is stated as follows:

$$
\begin{aligned}
& \underset{x}{\operatorname{minimize}}\|x\|_{\mathcal{A}} \\
& \text { subject to } x[\boldsymbol{f}]=x^{o}[\boldsymbol{f}], \boldsymbol{f} \in \mathcal{M},
\end{aligned}
$$

where $\mathcal{M}$ is the index set for measurements and $\mathcal{M} \subseteq \mathcal{N}$. Then we have the dual problem of (3.3) as follows:

$$
\begin{array}{r}
\underset{q}{\operatorname{maximize}}\left\langle q_{\mathcal{M}}, x_{\mathcal{M}}^{o}\right\rangle \\
\text { subject to }\|q\|_{\mathcal{A}}^{*} \leq 1, \\
q_{\mathcal{N} \backslash \mathcal{M}}=0,
\end{array}
$$

where $q_{\mathcal{M}}$ is the partial tensor of $q$ over the index set $\mathcal{M}$. Here, $\langle q, a(\boldsymbol{t}, 0)\rangle=\sum_{\boldsymbol{j}} q_{\boldsymbol{j}} e^{i 2 \pi \boldsymbol{t}^{T} \boldsymbol{j}}$, where $\boldsymbol{j}=\left\{j_{1}, j_{2}, \ldots, j_{d}\right\} \in$ $\mathcal{N}$, and $0 \leq j_{d} \leq n_{p}-1$ for $1 \leq p \leq d$. It is noteworthy that $\langle q, a(\boldsymbol{t}, 0)\rangle$ is a $d$-variate trigonometric polynomial with variables $\boldsymbol{t}$ or that is often termed the dual polynomial.

Let $\boldsymbol{m}=\left(m_{1}, m_{2}, \ldots, m_{d}\right)^{T}$, where $m_{p} \geq n_{p}-1, \forall p, 1 \leq p \leq d$, be a sum-of-squares relaxation degree vector and introduce the zero-padding extension $\tilde{q}$ of $q$ under $\boldsymbol{m}$, as follows. For $\boldsymbol{j}=\left(j_{1}, j_{2}, \ldots, j_{d}\right) \in \prod_{p=1}^{d}\left\{f \mid 0 \leq f \leq m_{p}\right\}, \tilde{q}$ is defined as follows:

$$
\tilde{q}_{j}= \begin{cases}q_{j} & \text { if } \boldsymbol{j} \in \mathcal{N} \\ 0 & \text { otherwise. }\end{cases}
$$

Then, for the vectorization of $\tilde{q}$, denoted by $\overrightarrow{\tilde{q}}$, we have the following equation for every $\boldsymbol{j}=\left(j_{1}, j_{2}, \ldots, j_{d}\right) \in$ $\prod_{p=1}^{d}\left\{f \mid 0 \leq f \leq m_{p}\right\}$ :

$$
\overrightarrow{\tilde{q}}_{u}=\tilde{q}_{j},
$$

where $u$ is the index for the vectorized variable represented as

$$
u=\left(\sum_{t=1}^{d}\left[j_{d-t+1}\left(\prod_{p=1}^{d-t}\left(m_{p}+1\right)\right)\right]\right)+1 .
$$

For each $1 \leq p \leq d$, we define the $\left(m_{p}+1\right) \times\left(m_{p}+1\right)$ Toeplitz matrix whose $k_{p}$-th diagonal elements are ones and zeros elsewhere as $\Theta_{k_{p}}$, where $-m_{p} \leq k_{p} \leq m_{p}$. We consider the diagonal of the matrix as the 0-th diagonal, and the uppermost diagonal as the $m_{p}$-th diagonal. Then, for a $d$-tuple $\boldsymbol{k}=\left(k_{1}, k_{2}, \ldots, k_{d}\right)$, where $-m_{p} \leq k_{p} \leq m_{p}$, $p=1,2, \ldots, d$, we define $\Theta_{\boldsymbol{k}}$ as follows:

$$
\Theta_{\boldsymbol{k}} \triangleq \Theta_{k_{d}} \otimes \ldots \otimes \Theta_{k_{1}}
$$


where $\otimes$ is the Kronecker product. From the Bounded Real Lemma for multivariate positive trigonometric polynomials, ${ }^{7}$ for $\|q\|_{\mathcal{A}}^{*}<1$, there exists a Hermitian matrix $Q \geq 0$ such that

$$
\delta_{\boldsymbol{k}}=\operatorname{Tr}\left[\Theta_{\boldsymbol{k}} Q\right], \boldsymbol{k} \in \mathcal{H}
$$

where $\mathcal{H}$ is a halfspace of $\prod_{p=1}^{d}\left\{k_{p} \mid-m_{p} \leq k_{p} \leq m_{p}\right\}, \delta_{\mathbf{0}}=1, \delta_{\boldsymbol{k}}=0$ if $\boldsymbol{k} \neq \mathbf{0}$, and

$$
\left[\begin{array}{cc}
Q & \overrightarrow{\tilde{q}} \\
\tilde{\tilde{q}}^{H} & 1
\end{array}\right] \geq 0
$$

Then, (3.4) is rewritten as follows:

$$
\begin{array}{cl}
\underset{q, Q}{\operatorname{maximize}} & \left\langle q_{\mathcal{M}}, x_{\mathcal{M}}^{o}\right\rangle_{\mathbb{R}} \\
\text { subject to } & \delta_{\boldsymbol{k}}=\operatorname{Tr}\left[\Theta_{\boldsymbol{k}} Q\right], \boldsymbol{k} \in \mathcal{H} \\
& {\left[\begin{array}{cc}
Q & \overrightarrow{\tilde{q}} \\
\overrightarrow{\tilde{q}}^{H} & 1
\end{array}\right] \geq 0,} \\
q_{\mathcal{N}} \backslash \mathcal{M} & =0,
\end{array}
$$

where $\tilde{q}$ is the extension of $q, \overrightarrow{\tilde{q}}$ is the vectorization of $\tilde{q}$, and $Q \in \mathbb{C}^{\left(\Pi_{p=1}^{d}\left(m_{p}+1\right)\right) \times\left(\Pi_{p=1}^{d}\left(m_{p}+1\right)\right)}$.

\section{PHASELESS ATOMIC NORM MINIMIZATION FOR MULTIDIMENSIONAL SIGNALS}

For some practical applications including optical fluorescence microscopy and X-ray crystallography, measuring phase information is much harder than measuring frequency magnitude information. When only magnitudes of frequency information are available to us, then (3.9) is not directly applicable. Hence, an SDP formulation of the ANM is required to recover a $2 \mathrm{D}$ or higher-dimensional signal in the continuous domain from only low frequency magnitude information. We call the SDP formulation as the phaseless ANM, simply PANM, for 2D or higher-dimensional signals. In this section, we extend the content of Section 3 and derive an SDP formulation that is applicable when only magnitude information is available.

The SDP formulation of the phaseless ANM is based on the following theorem which we prove next.

THEOREM 4.1. For a d-dimensional signal $x$ that can be written as in (2.2) and its vectorization $\vec{x} \in \mathbb{C}^{|\mathcal{N}|}$,

$$
\begin{aligned}
\|x\|_{\mathcal{A}}^{2} \geq \underset{z, l}{\operatorname{minimize}} \frac{1}{|\mathcal{N}|} z \operatorname{Tr}\left(T_{d}\right) \\
\text { subject to } U \triangleq\left[\begin{array}{ll}
T_{d} & \vec{x} \\
\vec{x}^{H} & z
\end{array}\right] \geq 0,
\end{aligned}
$$

where $T_{d} \in \mathbb{C}^{|\mathcal{N}| \times|\mathcal{N}|}$ is the d-level block Hermitian Toeplitz matrix defined as follows:

$$
T_{d} \triangleq \sum_{\boldsymbol{k} \in \mathcal{H}} \Theta_{\boldsymbol{k}} l_{\boldsymbol{k}}
$$

Here, $\Theta_{\boldsymbol{k}}$ is defined in (3.6) and $l_{\boldsymbol{k}}$ is an element of $\boldsymbol{l}$, which is the optimization variable in (4.1). Equality holds in (4.1) if the d-level block Toeplitz matrix $T_{d}$ can be decomposed as follows:

$$
T_{d}=V D V^{H}
$$

for the optimal solution $\boldsymbol{l}^{o}$, where $V$ is the Vandermonde matrix for a multidimensional signal having a $\left(\boldsymbol{t}_{j}, \phi_{j}\right)$ in the $j$-th column of $V, D$ is the diagonal matrix whose $j$-th diagonal element is $\left|d_{j}\right|$, and $\operatorname{rank}\left(T_{d}\right)=r<|\mathcal{N}|$.

Proof. For a given $x$, let us denote the optimal value of the optimization problem in (4.1) as $S D P(x)$. (4.1) can be proven by considering a feasible solution of the optimization problem in (4.1). For $\vec{x}=\sum_{j}\left|c_{j}\right| a\left(\boldsymbol{t}_{j}, \phi_{j}\right)$, 
let us choose a feasible solution for $z$ and $\boldsymbol{l}$ as follows: $\boldsymbol{l}$ such that $T_{d}=\sum_{j}\left|c_{j}\right| a\left(\boldsymbol{t}_{j}, \phi_{j}\right) a\left(\boldsymbol{t}_{j}, \phi_{j}\right)^{H}$ and $z=\sum_{j}\left|c_{j}\right|$. Then, we have

$$
\left[\begin{array}{cc}
T_{d} & \vec{x} \\
\vec{x}^{H} & z
\end{array}\right]=\sum_{j}\left|c_{j}\right|\left[\begin{array}{c}
a\left(\boldsymbol{t}_{j}, \phi_{j}\right) \\
1
\end{array}\right]\left[\begin{array}{c}
a\left(\boldsymbol{t}_{j}, \phi_{j}\right) \\
1
\end{array}\right]^{H} \geq 0 .
$$

For the feasible solution, the objective function value is $\frac{1}{|\mathcal{N}|} z \operatorname{Tr}(T d)=\left(\sum_{j}\left|c_{j}\right|\right)^{2}$, which is $\|x\|_{\mathcal{A}}^{2}$ by (3.1). Since $S D P(x)$ represents the minimum value among all feasible solutions, $\|x\|_{\mathcal{A}^{2}}^{2} \geq S D P(x)$.

The proof of the equality condition is shown by considering the relationship between $T_{d}$ and $\vec{x}$ in the positive semi-definite matrix of (4.1). Due to the positive semi-definiteness, $\vec{x}$ should be in the range space of $T_{d} ;$ namely, $\vec{x}=\sum_{j}\left|w_{j}\right| a\left(\boldsymbol{t}_{j}, \phi_{j}\right)$. Otherwise, we can always find a vector $v$ such that $v^{H} U v<0$, where $U$ is defined in (4.1). Because of positive semi-definiteness and $z>0$, the Schur complement of the matrix is also positive semi-definite, i.e.,

$$
V D V^{H}-\frac{1}{z} V w w^{H} V^{H} \geq 0 .
$$

When $V$ is full column rank, we can find a vector $v$ such that $V^{H} v=\operatorname{sign}(w)$, where $\operatorname{sign}(w)$ is a sign vector having $w_{j} /\left|w_{j}\right|$ as the $j$-th element. By using such $v$, we have

$$
\begin{aligned}
\operatorname{Tr}(D) & =v^{H} V D V^{H} v \\
& \geq \frac{1}{z} v^{H} V w w^{H} V^{H} v \\
& =\frac{1}{z} \operatorname{sign}(w)^{H} w w^{H} \operatorname{sign}(w) \\
& =\frac{1}{z}\left(\sum_{j}\left|w_{j}\right|\right)^{2},
\end{aligned}
$$

where the inequality is obtained from (4.5). Therefore, for the objective value of the optimization problem in (4.1), we have

$$
\frac{1}{|\mathcal{N}|} z \operatorname{Tr}\left(T_{d}\right)=\frac{1}{|\mathcal{N}|} z \operatorname{Tr}\left(V D V^{H}\right)=z \operatorname{Tr}(D) \geq\left(\sum_{j}\left|w_{j}\right|\right)^{2}=\|x\|_{\mathcal{A}}^{2}
$$

where the inequality is obtained from (4.6). With the first part showing that $\|x\|_{\mathcal{A}}^{2} \geq S D P(x)$, we conclude that if the Toeplitz matrix is decomposed as (4.3), then, $\|x\|_{\mathcal{A}}^{2}=S D P(x)$. If $z=0$, then, from the positive semi-definiteness of $U$, all the principal minor of $U$ should be non-negative. Therefore, all the elements in $x$ need to be 0 , and the equality in (4.1) still holds.

The existence of Vandermonde decomposition for a multidimensional signal as shown in (4.3) is studied in the previous research. ${ }^{8}$ According to the research, the Vandermonde decomposition for the $d$-level Toeplitz matrix $T_{d}$ exists if $\operatorname{rank}\left(T_{d}\right)<\min _{p} n_{p}$. When $\operatorname{rank}\left(T_{d}\right) \geq \min _{p} n_{p}$, a numerical method is proposed to find the decomposition in the research. ${ }^{8}$

For incorporating magnitude measurement in ANM, we propose the squared ANM as follows:

$$
\begin{aligned}
& \underset{x}{\operatorname{minimize}}\|x\|_{\mathcal{A}}^{2} \\
& \text { subject to } a_{j}(x)=a_{j}\left(x^{o}\right), j=1, \ldots, g .
\end{aligned}
$$

where $g$ is the number of measurements, and $a_{j}(x)$ is the magnitude mapping operator defined as $\left|\left\langle a_{j}, \vec{x}\right\rangle\right|$, where $a_{j}$ is a vector in the same dimension as $\vec{x}$. 
From Theorem 4.1, we relax (4.8) to the follows optimization problem:

$$
\begin{aligned}
& \underset{x, z, l}{\operatorname{minimize}} \frac{1}{|\mathcal{N}|} z \operatorname{Tr}\left(T_{d}\right) \\
& \text { subject to }\left[\begin{array}{ll}
T_{d} & \vec{x} \\
\vec{x}^{H} & z
\end{array}\right] \geq 0, \\
& a_{j}(x)=a_{j}\left(x^{o}\right), j=1, \ldots, g .
\end{aligned}
$$

Using the Schur complement to equivalently express the positive semi-definiteness constraint in (4.9), we have

$$
\begin{aligned}
& \underset{x, z, l}{\operatorname{minimize}} \frac{1}{|\mathcal{N}|} z \operatorname{Tr}\left(T_{d}\right) \\
& \text { subject to } T_{d}-\frac{1}{z} \vec{x} \vec{x}^{H} \geq 0, \\
& \qquad a_{j}(x)=a_{j}\left(x^{o}\right), j=1, \ldots, g .
\end{aligned}
$$

By multiplying $z$ to the first constraint, denoting $z T_{d}$ as $T_{d}^{\prime}$ and $\vec{x} \vec{x}^{H}$ as $X$, and relaxing the optimization problem by removing the $\operatorname{rank}(X)=1$ constraint, we have the following optimization problem:

$$
\begin{aligned}
\underset{X \geq 0, l^{\prime}}{\operatorname{minimize}} & \frac{1}{|\mathcal{N}|} \operatorname{Tr}\left(T_{d}^{\prime}\right) \\
\text { subject to } & T_{d}^{\prime}-X \geq 0, \\
& A_{j}(X)=A_{j}\left(X^{o}\right), j=1, \ldots,,
\end{aligned}
$$

where $T_{d}^{\prime} \triangleq \sum_{\boldsymbol{k} \in \mathcal{H}} \Theta_{\boldsymbol{k}} l_{\boldsymbol{k}}^{\prime}$, and $A_{j}(X)$ is a mapping function to the magnitude information; namely, $A_{j}(X)=$ $\operatorname{Tr}\left(A_{j} X\right)$, where $A_{j}=a_{j} a_{j}^{H}$. We call (4.11) the phaseless ANM (or simply PANM) for a multidimensional signal.

For concreteness, let us assume that only low frequency information is known and denoted by $X_{\mathcal{M}, \mathcal{M}} \in$ $\mathbb{C}^{|\mathcal{M}| \times|\mathcal{M}|}$, which represents the partial matrix $X$ over the index set $\mathcal{M}$ in columns and rows of the matrix $X \in \mathbb{C}^{|\mathcal{N}| \times|\mathcal{N}|}$. Then, by duality, we can have the following dual problem of (4.11) for a multidimensional signal:

$$
\begin{gathered}
\underset{Q \geq 0, W}{\operatorname{maximize}}\left\langle W_{\mathcal{M}, \mathcal{M}}, X_{\mathcal{M}, \mathcal{M}}^{o}\right\rangle_{\mathbb{R}} \\
\text { subject to } \delta_{\boldsymbol{k}}=\operatorname{Tr}\left[\Theta_{\boldsymbol{k}} Q\right], \boldsymbol{k} \in \mathcal{H} \\
\\
Q-W \geq 0, \\
W_{\mathcal{N} \backslash \mathcal{M}, \mathcal{N} \backslash \mathcal{M}}=0,
\end{gathered}
$$

where magnitude information is applied to $X_{\mathcal{M}, \mathcal{M}}^{o}$. In the following subsection, we describe a way to obtain $X_{\mathcal{M}, \mathcal{M}}^{o}$ from different linear combinations in magnitude.

\subsection{Using masks to obtain different linear combination magnitude information}

Different magnitude information in frequency domain can be obtained by using different linear combination $a_{j}(x)$ in (4.8). For example, from different linear combinations, we can have $|x[\boldsymbol{f}]|,|x[\boldsymbol{f}]+x[\boldsymbol{f}+\mathbf{1}]|$, and $\mid x[\boldsymbol{f}]-x[\boldsymbol{f}+\mathbf{1}]$. Motivated by the previous research, ${ }^{3,4,9}$ in this subsection, we introduce one way to obtain $X_{\mathcal{M}, \mathcal{M}}^{o}$ in (4.12) .

LEMma 4.2. Given magnitude measurements $\left|\vec{x}_{j}\right|=\left|\vec{x}_{j}^{o}\right|,\left|\vec{x}_{j}+\vec{x}_{j+1}\right|=\left|\vec{x}_{j}^{o}+\vec{x}_{j+1}^{o}\right|$ and $\left|\vec{x}_{j}-i \vec{x}_{j+1}\right|=\left|\vec{x}_{j}^{o}-i \vec{x}_{j+1}^{o}\right|$, $j=1, \ldots,|\mathcal{M}|$, where $\vec{x}_{j} \neq 0, j=1, \ldots,|\mathcal{M}|$, the positive semi-definite matrix $X_{\mathcal{M}, \mathcal{M}}$, which is the partial matrix of $X=\vec{x} \vec{x}^{H}$ over the index set $\mathcal{M}$ in columns and rows, is uniquely determined to $\vec{x}_{\mathcal{M}}^{o} \vec{x}_{\mathcal{M}}^{o}$.

Proof. Given magnitude measurements $\left|\vec{x}_{j}^{o}\right|,\left|\vec{x}_{j}^{o}+\vec{x}_{j+1}^{o}\right|$ and $\left|\vec{x}_{j}^{o}-i \vec{x}_{j+1}^{o}\right|, j=0,1, \ldots,|\mathcal{M}|-1$, we can have the diagonal, sub-diagonal, and super-diagonal of the matrix $X_{\mathcal{M}, \mathcal{M}}^{o}$ by solving linear equations together; namely, $X_{j, j}=\left|\vec{x}_{j}^{o}\right|^{2}$ for $j=1,2, \ldots,|\mathcal{M}|$, and $X_{j+1, j}=\vec{x}_{j+1}^{o} \vec{x}_{j}^{o *}$ and $X_{j, j+1}=\vec{x}_{j}^{o} \vec{x}_{j+1}^{o}{ }^{*}$ for $j=1,2, \ldots,|\mathcal{M}|-1$ are obtained. According to the non-negativeness of a Hermitian matrix, ${ }^{10}$ the Hermitian matrix is positive semi-definite if and 


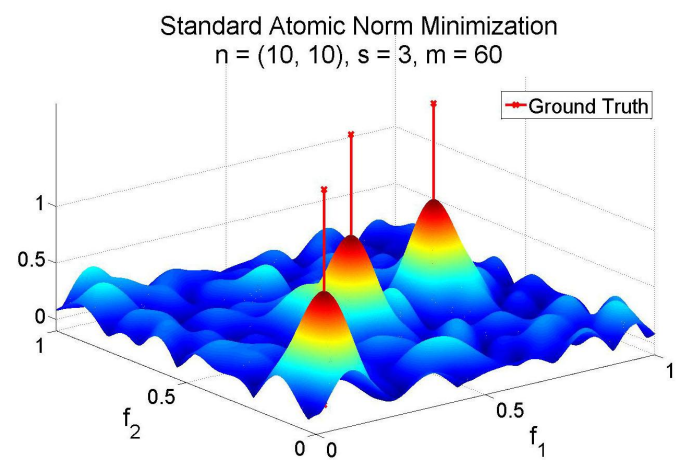

Figure 1. Dual polynomial obtained from the standard atomic norm minimization (3.9) with measurements including both phase and magnitude information.

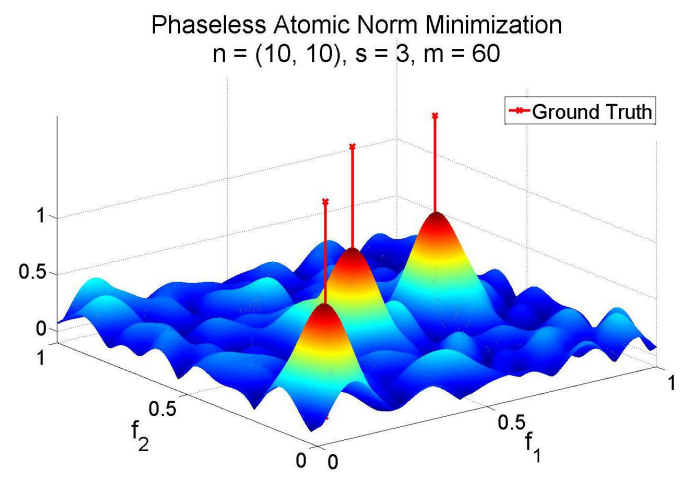

Figure 2. Dual polynomial obtained from the phaseless atomic norm minimization (4.12) with only magnitude measurements.

only if all of its principal minors are non-negative. Hence, all principal minors of $X$ including $X_{\mathcal{M}, \mathcal{M}}$ should be non-negative. Then, the lemma 4.2 can be proven by induction. When $|\mathcal{M}|=3$, the determinant of $X_{\mathcal{M}, \mathcal{M}}$ is

$$
\operatorname{det}\left(\left[\begin{array}{ccc}
\vec{x}_{1}^{o} \vec{x}_{1 *}^{o *} & \vec{x}_{1}^{o} \vec{x}_{2}^{o *} & X_{1,3} \\
\vec{x}_{2}^{o} \vec{x}_{1}^{o *} & \vec{x}_{2}^{o} \vec{x}_{2}^{o *} & \vec{x}_{2}^{o} \vec{x}_{3}^{o *} \\
X_{1,3}^{*} & \vec{x}_{3}^{o} \vec{x}_{2}^{o *} & \vec{x}_{3}^{o} \vec{x}_{3}^{o *}
\end{array}\right]\right)=-\left|\vec{x}_{2}^{o} X_{1,3}-\vec{x}_{1}^{o} \vec{x}_{2}^{o} \vec{x}_{3}^{o *}\right|^{2},
$$

where $X_{1,3}$ is unknown. To be $-\left|\vec{x}_{2}^{o} X_{1,3}-\vec{x}_{1}^{o} \vec{x}_{2}^{o} \vec{x}_{3}^{o *}\right|^{2} \geq 0, \vec{x}_{2}^{o} X_{1,3}-\vec{x}_{1}^{o} \vec{x}_{2}^{o} \vec{x}_{3}^{o *}=0$. Since $\vec{x}_{2}^{o} \neq 0, X_{1,3}$ is determined uniquely to $\vec{x}_{1}^{o} \vec{x}_{3}^{o *}$. When $|\mathcal{M}|=4$, the top-left $3 \times 3$ submatrix of $X_{\mathcal{M}, \mathcal{M}}$ and the bottom-right $3 \times 3$ submatrix of $X_{\mathcal{M}, \mathcal{M}}$ can be considered to determine $X_{1,3}$ and $X_{2,4}$ respectively. After figuring out $X_{1,3}$ and $X_{2,4}$, we can find $X_{1,4}$ by considering $3 \times 3$ principal submatrix of $X_{\mathcal{M}, \mathcal{M}}$ having $X_{1,4}$. In the similar way, when $|\mathcal{M}|=n \leq|\mathcal{N}|$, we can uniquely determine every unknown variables in $X_{\mathcal{M}, \mathcal{M}}$.

\section{NUMERICAL EXPERIMENTS}

We illustrate the efficacy of the proposed phaseless ANM with numerical simulations. We compare the simulation results with the standard ANM for 2D signal introduced in Section 3. For the 2D signal, we randomly generate a ground truth expressed as the sum of three Dirac delta functions, i.e., $s=3$, and the size of the signal dimension $n$ is $[10,10]^{T}$. The coefficient of each Dirac delta function, denoted by $c_{j}^{o}$ in $(2.2)$, is randomly generated, where the real part and the imaginary part of the coefficient followed $N(0,1)$. We use CVX $^{11}$ to solve (3.9) and (4.12) to obtain the dual polynomials.

For the magnitude measurements for PANM, we consider the case introduced in Lemma 4.2, where the number of measurements $|\mathcal{M}|=60$. The measurements used in ANM include both phase and magnitude information over the index set $\mathcal{M}$. Figure 1 and 2 demonstrate that the frequencies are successfully recovered from the dual polynomial obtained by solving (3.9) and (4.12) respectively. 


\section{CONCLUSION}

In this paper, we consider the recovery of a signal in $d$-dimension expressed as sum of delta Dirac functions whose possible locations are in the continuous spatial domain $[0,1]^{d}$. The proposed algorithm, called the phaseless atomic norm minimization for $d$-dimensional signals, demonstrates the possible signal recovery with only frequency magnitude information.

\section{REFERENCES}

[1] Möckl, L., Lamb, D. C., and Bräuchle, C., "Super-resolved fluorescence microscopy: Nobel prize in chemistry 2014 for eric betzig, stefan hell, and william e. moerner," Angewandte Chemie International Edition 53(51), 13972-13977 (2014).

[2] Born, M. and Wolf, E., [Principles of optics: electromagnetic theory of propagation, interference and diffraction of light], Elsevier (2013).

[3] Jaganathan, K., Saunderson, J., Fazei, M., Eldar, Y. C., and Hassibi, B., "Phaseless super-resolution using masks," in [Proceedings of IEEE International Conference on Acoustics, Speech and Signal Processing (ICASSP)], 4039-4043 (2016).

[4] Cho, M., Thrampoulidis, C., Xu, W., and Hassibi, B., "Phaseless super-resolution in the continuous domain," in [Proceedings of IEEE International Conference on Acoustics, Speech and Signal Processing $($ ICASSP)], (2017).

[5] Xu, W., Cai, J.-F., Mishra, K. V., Cho, M., and Kruger, A., "Precise semidefinite programming formulation of atomic norm minimization for recovering $d$-dimensional $(d \geq 2)$ off-the-grid frequencies," in [Proceedings of IEEE Information Theory and Applications Workshop (ITA)], 1-4 (2014).

[6] Chi, Y. and Chen, Y., "Compressive two-dimensional harmonic retrieval via atomic norm minimization," IEEE Transactions on Signal Processing 63(4), 1030-1042 (2015).

[7] Dumitrescu, B., [Positive trigonometric polynomials and signal processing applications], Springer (2007).

[8] Yang, Z., Xie, L., and Stoica, P., "Vandermonde decomposition of multilevel toeplitz matrices with application to multidimensional super-resolution," IEEE Transactions on Information Theory 62(6), 3685-3701 (2016).

[9] Candes, E. J., Strohmer, T., and Voroninski, V., "Phaselift: Exact and stable signal recovery from magnitude measurements via convex programming," Communications on Pure and Applied Mathematics 66(8), 1241$1274(2013)$.

[10] Meyer, C. D., [Matrix analysis and applied linear algebra], vol. 2, Siam (2000).

[11] Grant, M. and Boyd, S., "CVX: Matlab software for disciplined convex programming, version 2.0 beta." http://cvxr.com/cvx (Sept. 2012). 